\title{
Causas de cegiueira e baixa visão em crianças
}

\author{
Causes of blindness and low vision in children
}

Patrícia Ribeiro Brito ${ }^{(1)}$

Sílvia Veitzman ${ }^{(2)}$
Trabalho realizado no Departamento de Oftalmologia da Santa Casa de Misericórdia de São Paulo e tema livre no XIII Congresso Brasileiro de Prevenção da Cegueira e Reabilitação Visual.

(1) Médica residente do $3^{\circ}$ ano do Departamento de Oftalmologia da Santa Casa de Misericórdia de São Paulo.

(2) Doutora em Oftalmologia e Chefe do setor de Baixa Visão do Departamento de Oftalmologia da Santa Casa de Misericórdia de São Paulo.

Endereço para correspondência: Alameda das Catabas, L 1, Q. 11. Caminho das Árvores, Salvador (BA) CEP 41820-440.e-mail: brito@allways.com.br

\begin{tabular}{|c|}
\hline \hline RESUMO \\
\hline Objetivo: Identificar e analisar as principais causas preveníveis e \\
tratáveis da cegueira e baixa visão na infância. \\
Método: 174 crianças de 3 instituições de duas cidades brasileiras \\
(Salvador e São Paulo) foram examinadas e os dados de cada criança \\
foram registrados em protocolo padronizado pela OMS. \\
Resultados: De acordo com a OMS, encontrou-se cegueira ou baixa \\
visão em 82\% das crianças. Entre as moléstias preveníveis e tratáveis, a \\
retinopatia da prematuridade e o glaucoma foram as mais freqüentes. \\
Conclusão: O presente estudo piloto demonstrou características \\
similares às encontradas em outros países em desenvolvimento, onde \\
pelo menos $50 \%$ das doenças são preveníveis ou tratáveis com o atual \\
desenvolvimento científico.
\end{tabular}

Palavras-chave: Baixa visão (crianças); retinopatia da prematuridade; Glaucoma.

\section{INTRODUÇÃO}

A Organização Mundial de Saúde (OMS) relata que, anualmente, cerca de 500.000 crianças ficam cegas no mundo. Destas, 70 a $80 \%$ morrem durante os primeiros anos de vida, em conseqüência de doenças associadas ao seu comprometimento visual ${ }^{1}$.

Em 1992, a OMS estimava em 1,5 milhões o número de cegos menores do que 16 anos no mundo, $90 \%$ dos quais viviam em países em desenvolvimento ${ }^{1,2,3}$.

Estudos populacionais indicam baixa prevalência da cegueira infantil, de 0,2 a 0,3 por 1000 crianças em países desenvolvidos e de 1,0 a 1,5 por 1000 crianças em países em desenvolvimento. Já a prevalência de baixa visão é estimada como sendo três vezes maior, segundo o Banco de Dados Mundiais sobre a Cegueira da OMS ${ }^{2,4}$.

Recentemente (1996), a OMS adotou o conceito de anos de cegueira que resulta da multiplicação do número de cegos (ou portadores de baixa visão) pelo número de anos que a pessoa vive cega (ou com baixa visão). Esse conceito foi adotado para comparar a prevalência de diferentes problemas relacionados à deficiência visual. Assim, sendo a prevalência da cegueira e baixa visão infantil similar a da catarata senil, aquela também deve ser prioridade em Saúde Pública em oftalmologia na América Latina, ${ }^{2,4}$.

Com os conhecimentos médicos atuais, pelo menos $60 \%$ das causas de cegueira e severo comprometimento visual infantil são preveníveis ou tratáveis ${ }^{1,5}$.

Com base nestes e em outros dados, a OMS tem estimulado a implementação de programas de prevenção da perda visual ${ }^{6,7}$.

Conforme Temporini (1991), programas de saúde pública em oftalmologia devem priorizar ações relacionadas a prevenção de estados conducentes à cegueira e à incapacidade visual, a promoção de saúde 
ocular, a organização de assistência oftalmológica e a reabilitação de deficientes visuais ${ }^{8}$.

Para o planejamento e realização dessas ações, faz-se mister o conhecimento objetivo da realidade para a qual se destinam. Constata-se, portanto, a necessidade de se conhecer as causas de perda visual na população infantil, bem como sua distribuição geográfica, como requisito para o desenvolvimento desses programas ${ }^{1,7,8}$.

Os dados sobre as causas de comprometimento visual infantil nos países em desenvolvimento provêm, quase exclusivamente, da coleta de dados em escolas para cegos e hospitais especializados, pois, estudos populacionais são muito caros e demorados, o que torna difícil sua realização em países com escassos recursos na área de saúde ${ }^{9}$.

A literatura registra boa concordância entre estudos populacionais e amostrais em escolas para cegos e hospitais especializados das causas de cegueira e visão subnormal infantil ${ }^{1,2,5,9,10}$.

O objetivo do presente trabalho é identificar e analisar as principais causas preveníveis e/ou tratáveis de perda visual infantil em duas cidades brasileiras (São Paulo e Salvador).

\section{MATERIAL E MÉTODOS}

Foram avaliadas 174 crianças em três instituições, 76 do Instituto de Cegos "Padre Chico" de São Paulo, 38 do Instituto de Cegos da Bahia, na cidade de Salvador e 60 do setor de Baixa Visão do Departamento de Oftalmologia da Santa Casa de São Paulo, no período entre janeiro de 1996 e fevereiro de 1997. O estudo foi realizado com a aprovação das instituições envolvidas.

Oitenta e oito crianças $(50,6 \%)$ eram do sexo masculino e oitenta e seis $(49,4 \%)$ do sexo feminino. A idade média foi de 9 anos e 3 meses \pm 4 anos e 9 meses ( 1 mês a 15 anos).

O exame oftalmológico e os dados de cada criança foram registrados em um protocolo padronizado pela OMS, com a colaboração do Centro Internacional para Saúde Ocular (International Center for the Eye Health - ICEH), sediado em Londres (WHO/ PBL) (anexo) $)^{6,11}$.

Os protocolos foram preenchidos após exame das crianças ou análise dos prontuários médicos, conforme disponibilidade do Serviço.

\begin{tabular}{|c|c|}
\hline \multicolumn{2}{|c|}{ Classificação de comprometimento visual, segundo a OMS: } \\
\hline Classificação & Acuidade Visual * \\
\hline Sem comprometimento visual & $1,0 a>0,3$ \\
\hline Comprometimento visual moderado & $0,3 a>0,1$ \\
\hline Comprometimento visual severo & $0,1 \mathrm{a}>0,05$ \\
\hline Cegueira & $<0,05$ ou C.V. $<10^{\circ}$ \\
\hline
\end{tabular}

Com o intuito de facilitar a análise e a comparação com outros estudos sobre as causas de cegueira e visão subnormal (baixa visão), foram adotadas a definição de infância da UNICEF (período entre 0 e 15 anos) e a classificação para perda visual da OMS ${ }^{1,2}$.

Os dados foram computados e analisados no programa Paradox 3.5, que cruza informações, do Banco de Dados do WHO/PBL Eye Examination Record. ${ }^{6}$

\section{RESULTADOS}

Os resultados encontram-se nas tabelas 1 a 4 .

Seis crianças não apresentaram comprometimento visual, 142 apresentaram comprometimento visual de grau variável e 26 não puderam ser classificadas.

A principal localização anatômica da alteração ocular foi o bulbo ocular seguido pela retina e estruturas anatômicas relacionadas ao glaucoma.

O principal mecanismo patogênico foi desconhecido.

O glaucoma, a retinopatia da prematuridade e a rubéola foram as causas preveníveis e/ou tratáveis mais freqüentes no estudo (segundo critérios definidos no protocolo).

\section{DISCUSSÃO}

O grande número de crianças cegas $(63 \%)$ encontrado no estudo é explicado pelo fato da maioria delas pertencer a instituições para cegos.

Seis crianças possuíam déficit visual unilateral, sendo clas-

Tabela 1. Distribuição de crianças avaliadas em 3 instituições, de acordo com a classificação de comprometimento visual da OMS, no período entre jan/96 e fev/97

\begin{tabular}{|c|c|c|c|c|c|}
\hline \multirow[t]{2}{*}{ Classificação } & \multicolumn{3}{|c|}{ Instituições } & \multicolumn{2}{|c|}{ Total } \\
\hline & $\mathbf{A}$ & B & C & $\mathbf{N}$ & $\%$ \\
\hline Com comprometimento visual moderado & 0 & 0 & $16(26,7 \%)$ & 16 & $9,2 \%$ \\
\hline Cegueira & $72(94,7 \%)$ & $25(65,7 \%)$ & $12(20,0 \%)$ & 109 & $62,6 \%$ \\
\hline Inconclusivo & $3(4,0 \%)$ & $9(23,7 \%)$ & $14(23,3 \%)$ & 26 & $15,0 \%$ \\
\hline
\end{tabular}


Tabela 2. Distribuição de crianças cegas e com baixa visão avaliadas em 3 instituições, de acordo com a localização anatômica principal da alteração ocular responsável pela perda visual, no período entre Jan/96 e Fev/97.

\begin{tabular}{|lrr|}
\hline Localização & N & $\%$ \\
Bulbo Ocular & 39 & $27,5 \%$ \\
Córnea & 5 & $3,5 \%$ \\
Cristalino & 9 & $6,4 \%$ \\
Úvea & 5 & $3,5 \%$ \\
Retina & 42 & $29,5 \%$ \\
Nervo óptico & 11 & $7,7 \%$ \\
E. A. R. G. & 22 & $15,5 \%$ \\
Outros & 9 & $6,4 \%$ \\
Total & 142 & $100,0 \%$ \\
Fonte: 1) Instituto Padre Chico; 2) Instituto de Cegos da Bahia; 3) Santa Casa \\
de Misericórdia de São Paulo.; E.A.R.G.: Estruturas Anatômicas Relaciona- \\
das ao Glaucoma.
\end{tabular}

Tabela 3. Distribuição de crianças cegas e com baixa visão avaliadas em 3 instituições, de acordo com o mecanismo patogênico no período entre Jan/96 e Fev/97.

\begin{tabular}{|cccc|} 
Mec. Pat. & Período & N & $\%$ \\
Hereditário & & 15 & $10,5 \%$ \\
& Intra-uterino & 21 & $14,8 \%$ \\
Adquirido & Peri-natal & 20 & $14,1 \%$ \\
& Infância & 23 & $16,2 \%$ \\
Desconhecido & 63 & $44,4 \%$ \\
Total & 142 & $100,0 \%$ \\
Fonte: A) Instituto Padre Chico; B) Instituto de Cegos da Bahia; C)Santa \\
Casa de Misericórdia de São Paulo; Mec. Pat. = Mecanismo patogênico. \\
\hline
\end{tabular}

Fonte: A) Instituto Padre Chico; B) Instituto de Cegos da Bahia; C)Santa
Casa de Misericórdia de São Paulo; Mec. Pat. = Mecanismo patogênico. sificadas como sem comprometimento visual porque, conforme os critérios da OMS, a classificação leva em consideração a acuidade visual no melhor olho, com a melhor correção óptica.

Em vinte e seis crianças não foi possível aferir a acuidade visual com os métodos subjetivos comumente utilizados, pela pouca idade ou presença de outras deficiências, além da visual. Elas foram excluídas do estudo. Portanto, os resultados são referentes às 142 crianças que permaneceram no estudo.

Existe carência de publicações que mostrem quais as doenças mais prevalentes que determinam perda visual em nosso meio ${ }^{12}$. Dentre os diversos motivos que explicam esta realidade está a dificuldade de se obter um diagnóstico preciso de alguns pacientes que apresentam alterações em várias estruturas oculares e história clínica confusa ${ }^{13}$. Por isso, o conhecimento da localização anatômica principal da anomalia é útil para facilitar o diagnóstico diferencial ${ }^{6}$.

Alteração do bulbo ocular é definida como aumento ou redução do diâmetro do bulbo, como por exemplo microftalmia, phthisis bulbi, anoftalmia etc ${ }^{6,11}$.

Semelhantemente a outros estudos, a retina e o bulbo ocular foram os mais freqüentes locais de alteração ocular, com $29,5 \%$ e $27,5 \%$ dos casos, respectivamente. O fato é justificado por ser a retina afetada por variadas doenças, infecciosas ou não, e phthisis bulbi representar o estádio final de muitas moléstias $9,10,13,14$.

As estruturas anatômicas relacionadas ao glaucoma apresentaram elevada freqüência. Embora não se tenha explicação para o fato, possivelmente, fatores genéticos e infecciosos (como rubéola) estejam envolvidos.

$\mathrm{O}$ fator econômico está fortemente relacionado à etiologia

\begin{tabular}{|c|c|c|c|c|c|}
\hline Causas & $\overline{\mathbf{A}}$ & B & C & $\mathbf{N}$ & $\%$ \\
\hline Glaucoma & 18 & 6 & 2 & 26 & $18,3 \%$ \\
\hline Retinopatia da prematuridade & 12 & 5 & 0 & 17 & $12,0 \%$ \\
\hline Rubéola & 5 & 0 & 6 & 11 & $7,7 \%$ \\
\hline Neoplasia & 4 & 1 & 0 & 5 & $3,5 \%$ \\
\hline Encefalite / Meningite & 2 & 0 & 2 & 4 & $2,8 \%$ \\
\hline Sarampo & 1 & 1 & 0 & 2 & $1,4 \%$ \\
\hline Traumatismo & 2 & 0 & 0 & 2 & $1,4 \%$ \\
\hline Hipóxia neonatal & 0 & 0 & 1 & 1 & $0,7 \%$ \\
\hline Retinopatias & 6 & 1 & 5 & 12 & $8,5 \%$ \\
\hline Aniridia / Microftalmia & 4 & 1 & 2 & 7 & $4,9 \%$ \\
\hline Outros & 0 & 0 & 3 & 3 & $2,2 \%$ \\
\hline Total geral & 73 & 27 & 42 & 142 & $100 \%$ \\
\hline
\end{tabular}


da perda visual ${ }^{15}$. Nos países em desenvolvimento, em especial os africanos e asiáticos, os agentes infecciosos são os principais causadores de doenças oculares. Provavelmente, esses números são muito maiores do que deixam entrever os dados disponíveis, pois esses agentes também são responsáveis pela alta taxa de mortalidade infantil desses países $1,2,3,9,15$.

As moléstias hereditárias apresentam-se com freqüência maior nos países desenvolvidos, onde os agentes infecciosos influem pouco na perda visual. Nestes países, a melhor qualidade de vida da população e o amplo acesso aos bons serviços de saúde são a explicação para esse fato.

Neste trabalho, 10,5 \% das doenças foram de transmissão hereditária e $16,8 \%$ de etiologia infecciosa. Em 44,4\% dos casos, o mecanismo patogênico da doença foi desconhecido, como por exemplo o glaucoma congênito, a catarata congênita sem história familiar e com sorologias negativas.

A retinopatia da prematuridade está entre as primeiras causas de cegueira infantil, tanto em países desenvolvidos quanto em países em desenvolvimento. Naqueles, a melhor assistência neonatal permite a sobrevida de muitos recémnascidos com baixo peso e prematuros, que formam o principal grupo de risco para a ocorrência da doença e suas complicações, apesar do acompanhamento periódico e intervenção imediata quando necessária. Nas duas cidades estudadas, e em outras cidades de países em desenvolvimento, a dificuldade para adequada assistência pré-natal, acompanhamentos periódicos e tratamento explicam a elevada ocorrência.

Neste estudo, as causas mais freqüentes foram o glaucoma congênito, a retinopatia da prematuridade, a rubéola, a catarata congênita e a toxoplasmose congênita. Estes dados são diferentes dos da OMS, que identifica como causas importantes de cegueira no Brasil a hipovitaminose $\mathrm{A}$, a oncocercose, o sarampo e o tracoma ${ }^{5,16,17}$.

Kara-José et al., em estudo retrospectivo sobre as causas de comprometimento visual em crianças, realizado em duas cidades brasileiras (São Paulo e Goiânia) na década passada, já apontavam a elevada freqüência do glaucoma congênito, da catarata congênita e da retinopatia e afirmavam não serem a hipovitaminose A, o tracoma e a oncocercose causas importantes de deficiência visual em nosso meio ${ }^{16}$.

O presente estudo, o primeiro realizado no Brasil utilizando o protocolo de causas de cegueira e baixa visão da OMS ${ }^{4,17}$, demonstrou que $59 \%$ das doenças oculares são preveníveis ou tratáveis com o atual desenvolvimento científico, dados similares a outros países em desenvolvimento.

O Brasil, com suas dimensões continentais e diferenças econômicas e culturais nos diversos estados, exige avaliação ampla sobre o tema para o estabelecimento de programas de prevenção das causas de comprometimento visual infantil. Neste estudo piloto, propomos a utilização do protocolo da OMS como forma de padronizar os conceitos e avaliações, pois, além da necessidade individual e comunitária, temos como obrigação ética a prevenção.

\section{SUMMARY}

Purpose: To identify and analyze the major preventable and treatable causes of loss of vision in children.

Methods: Children (174) from three institutions were examined in two Brazilians cities (Salvador and São Paulo) and data were recorded acording to the WHO protocol. Results: According to WHO visual impairment was found in $82 \%$ of the children. The most frequent preventable and treatable ocular diseases were retinopathy of prematurity and glaucoma. Conclusions: This pilot study shows similar characteristics to other developing countries, where at least $50 \%$ of ocular diseases are preventable or treatable with today's knowledge.

Keywords: Low vision (children); Retinopathy of prematurity; Glaucoma.

\section{REFERÊNCIAS BIBLIOGRÁFICAS}

1. Foster A, Gilbert C. Epidemiology of Childhood Blindness. Eye 1992;6:173-6.

2. Bischh F. Análisis epidemiológico de la ceguera. Arch Chil Oftal 1995;52(1):55-70.

3. Thylefors B. Situación Mundial de la Ceguera. Bol of Sanit Panam 1995; 119(2):179-82.

4. Foster A. Curso de Epidemiologia de Causas de Cegueira. Fundación Oftalmologica de Santander. Bucaramanga-Colombia. Maio, 1996.

5. Oliveira PR. Causas de Cegueira na Infância. Arq Bras Oftalmol 1992;55(4):174-5.

6. Gilbert C, Foster A. Childhood Blindness: a new form for recording causes of visual loss in children. Bulletin of the World Health Organisation 1993;71(5):485-9.

7. Temporini ER, Kara-José N. Níveis de prevenção de problemas oftalmológicos: propostas de investigação. Arq Bras Oftalmol 1995;58(3):189-92.

8. Temporini ER. Pesquisa de oftalmologia em Saúde Pública: considerações metodológicas sobre fatores humanos. Arq Bras Oftalmol 1991;54(6):279-81.

9. Gilbert CE, Canovas R, Canovas RK, Foster A. Causes of blindness and severe visual impairment in children in Chile. Developmental Medicine and Child Neurology 1994;36:326-33.

10. Moreira ATR, Moreira CA, Arana J. Causas de cegueira no Instituto de Cegos do Paraná. Arq Bras Oftalmol 1991;54(6):275-7.

11. Cunha RP. Resumo e tradução do formulário elaborado pelo Centro Internacional para Saúde Ocular e pela Organização Mundial da saúde para registro de crianças com cegueira e visão subnormal. Arq Bras Oftalmol 1993;56(5):284-8.

12. Leal DB, Tavares SS, Ventura LO, Florêncio T. Atendimento a portadores de visão subnormal: estudo retrospectivo de 317 casos. Arq Bras Oftalmol 1995;58(6):439-42.

13. Tartarella MB, Nakano K, Castro CTM, Martins APM. Visão subnormal em crianças. Arq Bras Oftalmol 1991;54(5):221-4.

14. Gilbert C, Foster A. Causes of blindness in children attending four schools for the blind in Thailand and The Phlippines. International Ophthalmology 1993;17:229-34.

15. Esteves JF, Domingues CG, Borges LPK, Skolaude PBV, Bortolomiol L, Muxfeldt RA, Bisol T, De Souza CA, Marsico J, Fior O. Prevalência e causas de cegueira em bairro de Porto Alegre. Arq Bras Oftalmol 1996;59(3):244-7.

16. Kara-José N, Almeida GV, Arieta CEL, Araújo JS, Becgara SJ, Oliveira PR. Causas de deficiência visual em crianças. Bol of Sanit Panam 1994;97(5): 405-12.

17. Simmons WK. Xerophthalmia and blindness in Northeast Brazil. The American Journal of Clinical Nutricion 1976;29:116-22. 


\section{G. Refraçāo:}

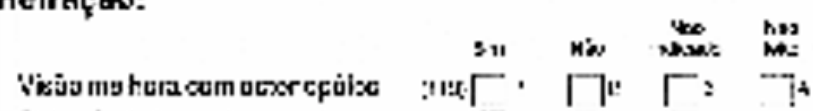

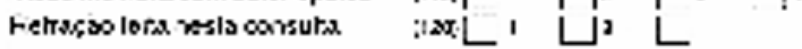
उ० आเ।

1: AY contalds p! longo:

loshe cods clo sepuracsmanle, deposs junles

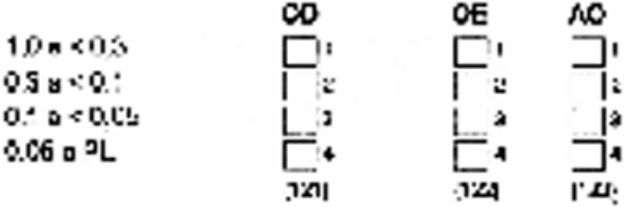

Issecïiqub la ves co tetcres o di

ch: a.d:

C't

AV

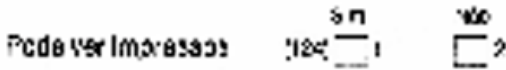

H. Examc ocular - Etiologla de perda visual

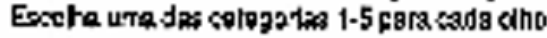

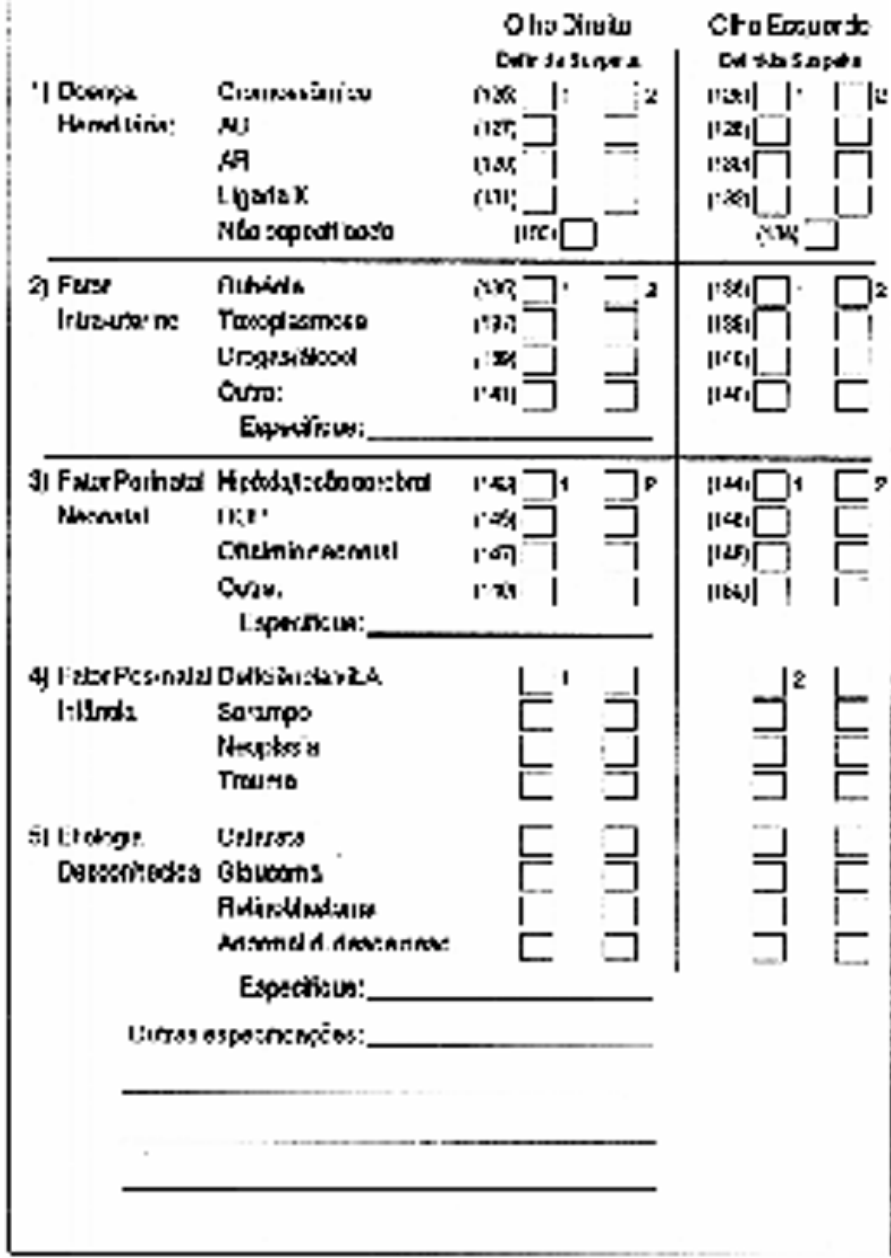

Prinoipol etiologia dn perdn viзual para a criança:

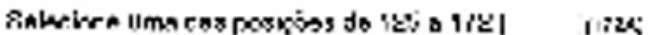

\section{Conduta necoeceśris}

1JÓptho:

Horhume

Ruly;in: mass tade

Station

Noxl5oYSH

Nomp: 2x:B.

:

$7 x_{1}$

:

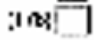

: Mediealíinumglea

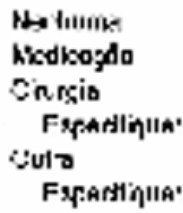

(1)!

(1)

is:

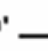

\section{J. Prognásliea visual}

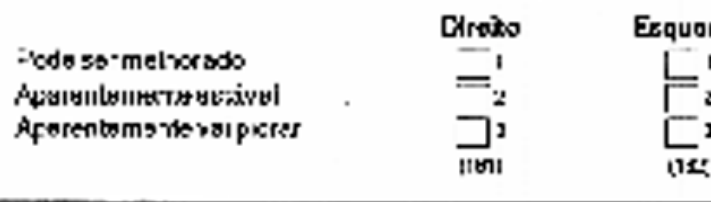

\section{KEducaçåo}

1) Facoin alin|

Expoul poracozra

Cypec al pard mubplos doikertas

Filikgolsintagmin

Nontrame

Unts

Esputalia,

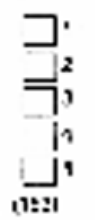

Z) Reoomendoçdeo

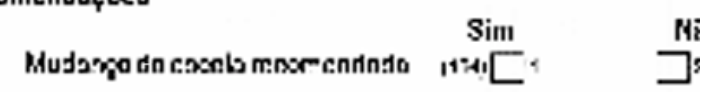
Fapark.

\section{Diagnóstico}

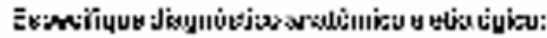

oroCirclı:

(Imony)

\section{Examinador:}

Examinzidofcr.

Dous: $\prod_{\text {ils: }}^{\text {indsi }}$ 


\section{G.Refração:}

Sim $\quad$ Não $\quad \begin{gathered}\text { Não } \\ \text { indicado }\end{gathered} \quad \begin{gathered}\text { Náo } \\ \text { feito }\end{gathered}$ Refração teita nesta consulta

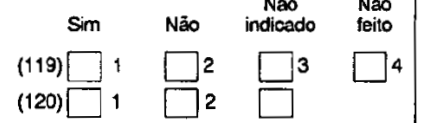
Se SIM

1) AV corrigida p/longe:

Teste cada olho separadamente, depois juntos

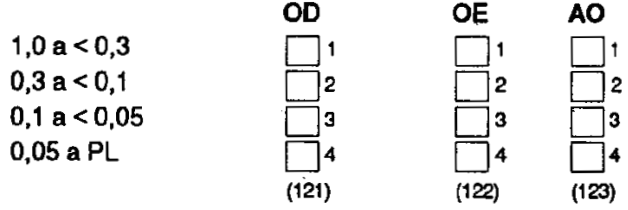

Especifique lentes corretoras e AV

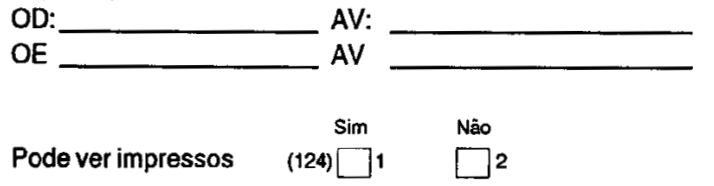

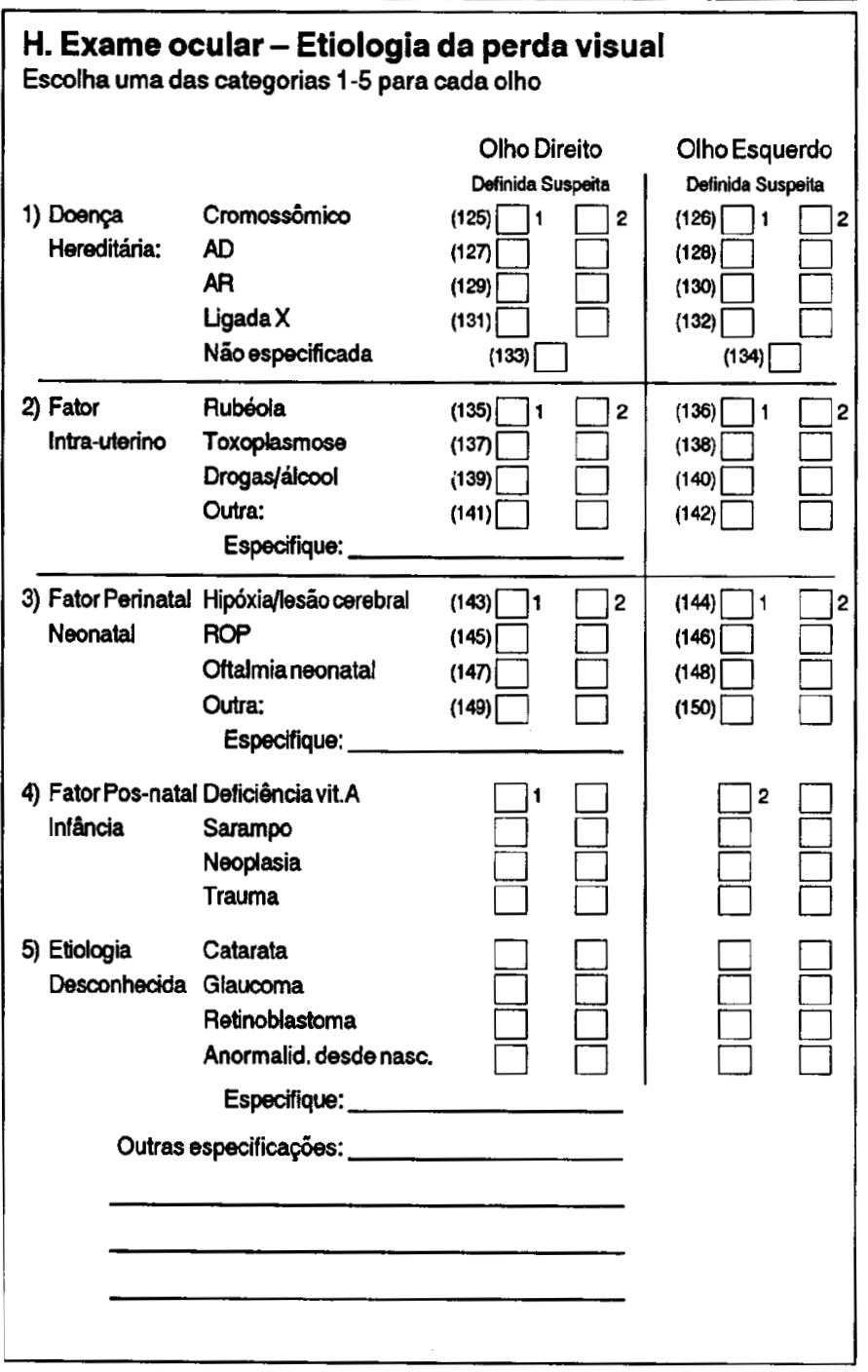

Principal etiologia da perda visual para a criança:

Selecione uma das posiçōes de 125 a 172 ] J(172A)

\section{Conduta necessária}

1) Óptica:

$\begin{array}{lc}\text { Nentuma } & \text { Marque oaplicável } \\ \text { Refração mais tarce } & (173) \square \\ \text { Óculos } & (174) \square \\ \text { Auxilio VSN } & (175) \square \\ & (176) \square\end{array}$

2) Médico/Cirúrgico

\begin{tabular}{|c|c|}
\hline Nenhuma & (177) \\
\hline Medicação & (178) [ \\
\hline $\begin{array}{l}\text { Cirurgia } \\
\text { Especifique: }\end{array}$ & (179) [ \\
\hline $\begin{array}{l}\text { Outra } \\
\quad \text { Especifique: }\end{array}$ & $(180)[$ \\
\hline
\end{tabular}

\section{J. Prognóstico visual}

Marque apenas um quadrado
para cada olho
Pode ser melhorado
Aparentemente estável

\section{K. Educação}

\section{1) Escola atual}

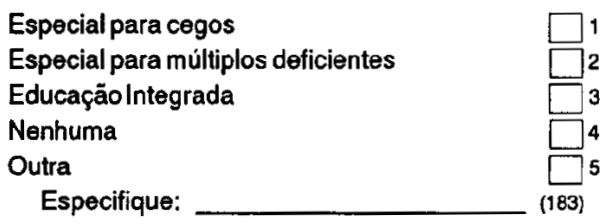

2) Recomendaçōes

Mudança da escola recomendada $\quad$ (184) $\square$ N
Especifique:

\section{Diagnóstico}

Especifique diagnóstico anatômico e eticlógico:

Otho Direito:

Olhoesquerdo:

\section{Examinador:}

Examinadopor:

Data: $\quad$ (mês) (ano)

